University of Nebraska - Lincoln DigitalCommons@University of Nebraska - Lincoln

Uniformed Services University of the Health

Sciences

U.S. Department of Defense

2014

\title{
Three-dimensional joint reaction forces and moments at the low back during over-ground walking in persons with unilateral lower-extremity amputation
}

Brad Hendershoot

Walter Reed National Military Medical Center, Uniformed Services University of the Health Sciences, bradford.d.hendershot.ctr@health.mil

ErikJ. Wolf

Walter Reed National Military Medical Center,

Follow this and additional works at: http://digitalcommons.unl.edu/usuhs

Hendershoot, Brad and Wolf, Erik J., "Three-dimensional joint reaction forces and moments at the low back during over-ground walking in persons with unilateral lower-extremity amputation" (2014). Uniformed Services University of the Health Sciences. 130. http://digitalcommons.unl.edu/usuhs/130

This Article is brought to you for free and open access by the U.S. Department of Defense at DigitalCommons@University of Nebraska - Lincoln. It has been accepted for inclusion in Uniformed Services University of the Health Sciences by an authorized administrator of DigitalCommons@University of Nebraska - Lincoln. 


\title{
Three-dimensional joint reaction forces and moments at the low back during over-ground walking in persons with unilateral lower-extremity amputation
}

\author{
Brad D. Hendershot ${ }^{\mathrm{a}, \mathrm{b}, *}$, Erik J. Wolf ${ }^{\mathrm{a}, \mathrm{c}}$ \\ a Department of Rehabilitation, Walter Reed National Military Medical Center, Bethesda, MD 20889, USA \\ b Center for Rehabilitation Sciences Research, Department of Physical Medicine and Rehabilitation, Uniformed Services University of the Health Sciences, Bethesda, MD 20814, USA \\ c DOD - VA Extremity Trauma and Amputation Center of Excellence, Walter Reed National Military Medical Center, Bethesda, MD 20889, USA
}

\section{A R T I C L E I N F O}

\section{Article history:}

Received 15 August 2013

Accepted 5 December 2013

\section{Keywords:}

Amputation

Lumbosacral

Gait

Inverse dynamics

Low back pain

Biomechanics

\begin{abstract}
A B S T R A C T
Background: Abnormal mechanics of locomotion following lower-extremity amputation are associated with increases in trunk motion, which in turn may alter loads at the low back due to changes in inertial and gravitational demands on the spine and surrounding trunk musculature.

Methods: Over-ground gait data were retrospectively compiled from two groups walking at similar self-selected speeds $(\sim 1.35 \mathrm{~m} / \mathrm{s}): 40$ males with unilateral lower-extremity amputation (20 transtibial, 20 transfemoral) and 20 able-bodied male controls. Three-dimensional joint reaction forces and moments at the low back (L5/S1 spinal level) were calculated using top-down and bottom-up approaches. Peak values and the timings of these were determined and compared between and within (bilaterally) groups, and secondarily between approaches.

Findings: Peak laterally-directed joint reaction forces and lateral bend moments increased with increasing level of amputation, and were respectively $83 \%$ and $41 \%$ larger in prosthetic vs. intact stance among persons with transfemoral amputation. Peak anteriorly-directed reaction forces and extension moments were $31 \%$ and $55 \%$ larger, respectively, among persons with transtibial amputation compared to controls. Peak vertical reaction forces and axial twist moments were similar between and within groups. Peak joint reaction forces and moments were larger (3-14\%), and the respective timing of these sooner (11-62 ms), from the bottom-up vs. top-down approach.

Interpretation: Increased and asymmetric peak reaction forces and moments at the low back among persons with unilateral lower-extremity amputation, particularly in the frontal plane, suggest potential mechanistic pathways through which repeated exposure to altered trunk motion and spinal loading may contribute to low-back injury risk among persons with lower-extremity amputation.
\end{abstract}

(c) 2013 Elsevier Ltd. All rights reserved.

\section{Introduction}

Altered and asymmetric gait and movement are common among persons with lower-extremity amputation (LEA; Sagawa et al., 2011). Such changes in the mechanics of locomotion have been associated with the development of secondary physical conditions and pain (Gailey et al., 2008). Low back pain (LBP), in particular, represents a frequent and debilitating impairment in this population that can often limit physical performance and reduce quality of life (Ehde et al., 2001; Taghipour et al., 2009). Moreover, recent and projected increases in the number of persons with LEA, resulting from traumatic injuries sustained during times of war (Reiber et al., 2010) and complications of vascular disease (Ziegler-Graham et al., 2008), further highlight the importance of understanding the underlying mechanisms linking LEA

\footnotetext{
* Corresponding author at: Walter Reed National Military Medical Center, Americ Building No. 19, Room B322, 8901 Wisconsin Ave, Bethesda, MD 20889, USA.

E-mail address: bradford.d.hendershot.ctr@health.mil (B.D. Hendershot).
}

and LBP; yet, such mechanisms are still unclear. Though most LBP remains idiopathic, physical (biomechanical) risk factors appear to play a more important role in this population.

Increased spinal loads have been identified as an important proximate cause of LBP (Kumar, 2001; McGill, 2007). Mechanical loads among tissues in/surrounding the spine are influenced by forces arising from gravity, inertia, and externally applied loads, as well as internal forces produced by ligaments and muscle contractions. Of particular interest here, the trunk ( + head and arms) accounts for nearly two thirds of total body mass (Winter, 1990), and as such even small displacements of the trunk center of mass can substantially alter muscular demands and joint reaction loads throughout the body (Gillet et al., 2003). For persons with unilateral LEA, increased and asymmetric trunk movements during locomotion have been observed (Cappozzo et al., 1982; Goujon-Pillet et al., 2008; Jaegers et al., 1995; Michaud et al., 2000; Tura et al., 2010), and which have been suggested to result from a neuromuscular/movement strategy that uses trunk weight/ inertia to assist with forward progression and/or stabilizing the body. 
Hence, altered trunk motions during gait among persons with LEA may result in spinal loading patterns distinct from able-bodied individuals, due to changes in the inertial and gravitational demands on the spine and surrounding trunk musculature. Despite the aforementioned alterations in trunk kinematics with LEA, there exists only limited preliminary work indicating altered spinal loads during gait in persons with LEA (Cappozzo and Gazzani, 1983).

Linked-segment models are a common non-invasive method for estimating reactive joint loads during movement (e.g., Kingma et al., 2001; MacKinnon and Winter, 1993). In able-bodied individuals, these models have been used to quantify net reaction forces and moments at the low back during occupational tasks, such as lifting (Kingma et al., 1996; Plamondon et al., 1996), as well as during walking and running (Callaghan et al., 1999; Cappozzo, 1983; Khoo et al., 1995; Seay et al., 2008). These models and analyses typically originate either at the head and work down (i.e., top-down approach), or from the feet and work up (i.e., bottom-up approach). Despite inherent limitations of linkedsegment models (Winter, 1990), and specific criticisms for the application of top-down and bottom-up approaches to locomotion (Callaghan et al., 1999; Riemer et al., 2008; Seay et al., 2008), these models have been extensively validated for estimating forces and net moments acting at the low back during a variety of tasks (Iino and Kojima, 2012; Kingma et al., 1996; Kingma et al., 2001). The primary goal of this study was to investigate triaxial joint reaction forces and moments at the low back (L5/S1 spinal level) in persons with unilateral LEA during over-ground walking. We hypothesized that persons with LEA would have increased and asymmetric lumbosacral joint loads compared to uninjured controls, due to changes in the gravitational and inertial contributions resulting from increased trunk motion. We further hypothesized that these changes would be larger for persons with transfemoral vs. transtibial amputation, as larger trunk motions are generally associated with a higher level of amputation. As a secondary goal, we also explored differences in the approach (bottomup vs. top-down) used to calculate L5/S1 reaction forces and moments in this population.

\section{Methods}

\subsection{Participants}

Data were retrospectively compiled and analyzed from 40 males with unilateral LEA - 20 transtibial (TTA) and 20 transfemoral (TFA) - and 20 male able-bodied controls (CTRL) that had previously completed gait evaluations (Table 1). All amputations were a result of traumatic injuries, and the mean (SD) duration since amputation was 2.6 (1.3) years. Inclusion criteria for the participants with LEA included: (1) unilateral transtibial or transfemoral amputation with no contralateral functional impairments, (2) regular (daily) use of a prosthetic device ( $\geq 1$ year post-amputation), (3) independent ambulation without the use of an upper-extremity assistive device (e.g., cane, crutches, walker), and (4) having no other underlying musculoskeletal or neurologic conditions (excluding amputation) that may affect gait or balance. Also, participants (in all groups) were only included if their self-selected walking velocity was between 1.25 and $1.40 \mathrm{~m} / \mathrm{s}$, as walking speed influences kinetic and kinematic biomechanical measures (Cheng et al., 1998). These retrospective analyses were approved by the local Institutional Review Board.

Table 1

Mean (SD) participant characteristics for the control ("CTRL"), transtibial ("TTA"), and transfemoral ("TFA") groups. Time since amputation ("Time") is also indicated.

\begin{tabular}{llcc}
\hline & CTRL $(n=20)$ & TTA $(n=20)$ & TFA $(n=20)$ \\
\hline Age $($ year $)$ & $28.1(4.8)$ & $27.7(6.5)$ & $29.2(6.7)$ \\
Stature $(\mathrm{cm})$ & $181.0(6.1)$ & $180.4(5.0)$ & $176.2(6.7)$ \\
Body mass $(\mathrm{kg})$ & $83.9(8.6)$ & $87.2(13.3)$ & $80.6(12.2)$ \\
Time (year) & - & $1.8(1.5)$ & $3.1(1.4)$ \\
\hline
\end{tabular}

\subsection{Experimental Procedures}

Participants walked at their self-selected velocity across a $15 \mathrm{~m}$ level walkway. During walking trials, full-body kinematics were tracked $(120 \mathrm{~Hz})$ via retro-reflective markers using a 23-camera motion capture system (Vicon, MX F40, Oxford, UK). Markers were placed in the mid-sagittal plane over the sacrum (S1), T10, and C7 spinous processes, sternal notch, and xiphoid; and bilaterally over the acromion, ASIS, PSIS, and lower extremities (modified Cleveland Clinic marker set). Ground reaction forces were sampled $(1200 \mathrm{~Hz})$ from four force platforms (AMTI, OR6-7-2000, Watertown, MA, USA) centrally located and embedded in the walkway. Raw marker and force platform data were low-pass filtered using a fourth-order, bidirectional, Butterworth filter with a $6 \mathrm{~Hz}$ and $50 \mathrm{~Hz}$ cutoff frequency, respectively.

\subsection{Dependent measures and analyses}

Joint reaction forces and moments at the low back (L5/S1) were estimated using a three-dimensional linked-segment model in Visual3D (C-Motion Inc., Germantown, MD, USA), which included fifteen segments defined by the markers: bilateral feet, shanks, and thighs; a pelvis; a trunk; bilateral upper arms, lower arms, and hands; and a head. The trunk was considered a single rigid segment, defined proximally by the acromia, C7, and sternal notch, and attached distally to the pelvis at the lumbosacral (L5/S1) joint (cf., Kingma et al., 1996). The location of the L5/S1 joint was estimated using the bony pelvis landmarks (ASIS, PSIS, and S1) and scaled to pelvis width (right ASIS to left ASIS; Reed et al., 1999). Segment inertial and anthropometric properties were calculated according to the regression equations of Hanavan and Dempster, respectively, and the residual limbs/prostheses were modeled using parameters identical to participants' intact segments. Three-dimensional trunk kinematics (and angular velocities/accelerations; Kinzel et al., 1972) were calculated, relative to the pelvis, using an X-Y-Z (sagittalcoronal-transverse) rotation sequence.

Two modeling approaches were then used to estimate reaction forces and moments at the L5/S1 joint: 1) a bottom-up approach, commencing at the feet and working up, and 2) a top-down approach, commencing at the head (arms) and working down. In both approaches, the analyses finished at the L5/S1 joint. For the bottom-up approach, ground reaction forces from both lower extremities were required as inputs into the model. Thus, data from multiple walking trials were identified which contained 5 "clean" strides; clean strides were defined by both the right and left foot remaining completely inside the boundary of two consecutive force platforms during successive initial contacts of the same foot. Although ground reaction forces are not required for the top-down model, the same five "clean" strides were used in subsequent analyses for both approaches. No verbal instructions were initially given that would indicate consecutive clean foot strikes were required, as these would likely influence gait. To resolve the kinetics at the low back in a more clinically relevant reference frame, an anatomical coordinate system was defined by aligning the pelvis with a marker projected directly below the L5/S1 joint at the mean height of the two hip joint centers (cf. Seay et al., 2008). Net lumbosacral forces and moments were resolved with respect to the trunk local coordinate system, and normalized to body mass and the product of body mass $*$ stature, respectively.

Following the calculation of L5/S1 reaction forces and moments using both approaches, all data were time-normalized to a stride (100\% gait cycle). Strides were defined from right heel strike to subsequent right heel strike for able-bodied controls, and from intact heel strike to subsequent intact heel strike for persons with LEA. Peak values of the three components of joint reaction force and moment were extracted from each side (i.e., left/right stance for controls, and intact/prosthetic stance for the TTA and TFA groups). The timings of these peaks, relative to ipsilateral heel strike, were also determined. Temporal-spatial parameters were also calculated (per side, where relevant), including walking speed, step length, and the duration of stance and swing. 
Two-way mixed-factor analyses of variance (ANOVAs) were then used to compare the dependent measures (from the top-down approach) between and within (bilaterally) groups. Where the effect of group or side was significant, a set of post-hoc contrasts (with a Scheffé correction; $P_{\text {critical }}=0.0043$ ) was used to determine: 1 ) overall differences between groups, 2) bilateral differences within groups, and 3) differences within limb type (e.g., intact and prosthetic) between TTA and TFA groups. When comparing peak values between sides, only the magnitude was used, to remove the directional component (e.g., positive lateral forces were directed to the right, and negative lateral forces to the left). For comparisons between top-down and bottomup inverse dynamics approaches, model type and all first-order interactions (i.e., with group and side) were added to the ANOVA model, and similar post-hoc contrasts were made to identify whether both models predicted similar trends between and within groups. All statistical analyses were performed using JMP (version 10, SAS Institute Inc., Cary, NC, USA). Summary values are reported as means (SD).

\section{Results}

\subsection{Temporal-spatial parameters}

Self-selected walking velocities were 1.35 (0.05), 1.35 (0.06), and $1.34(0.05) \mathrm{m} / \mathrm{s}$ for the CTRL, TTA, and TFA groups, respectively; these were not significantly different between groups $(P=0.91)$. Among participants with TTA and TFA, the prosthetic limb (relative to intact) was characterized by a longer step length, shorter stance duration, and longer swing duration (Table 2).

\subsection{Trunk kinematics}

In the frontal plane, the trunk flexed laterally towards the support leg, reaching a peak during single-limb stance (Fig. 1A). Peak trunk lateral flexion was bilaterally similar $(P=0.14)$ among controls [left $=4.1(1.7)^{\circ}$ vs. right $\left.=4.8(1.1)^{\circ}\right]$, but significantly larger $(P<0.0001)$ in prosthetic vs. intact limb stance among persons with TTA $\left[6.7(2.3)^{\circ}\right.$ vs. $\left.5.1(2.1)^{\circ}\right]$ and TFA $\left[9.1(2.3)^{\circ}\right.$ vs. $\left.5.5(1.1)^{\circ}\right]$. Trunk lateral excursions increased $(P<0.001)$ with increasing level of amputation, with respective values of 9.0 (2.2), 11.8 (3.8), and $14.7(2.2)^{\circ}$ for the CTRL, TTA, and TFA groups. In the sagittal plane, the trunk flexed forward following heel strike, extending prior to subsequent heel strike (Fig. 1B). Trunk flexion-extension excursions were larger $(P=0.001)$ among participants with TFA $\left[5.8(2.3)^{\circ}\right]$ compared to controls [2.1 $\left.(0.6)^{\circ}\right]$ and participants with TTA $\left[1.8(0.6)^{\circ}\right]$. In the transverse plane, the trunk rotated towards the support leg, with peak rotations occurring around heel strike (Fig. 1C). Peak axial twist motions were bilaterally similar $(P>0.22)$ in all three groups. Trunk axial rotational excursions tended $(P=0.09)$ to increase with increasing level of amputation, with respective values of $13.2(3.7), 14.7$ (2.7), and $15.4(4.7)^{\circ}$ for the CTRL, TTA, and TFA groups.

\subsection{Joint reaction forces and moments}

Lateral joint reaction forces were directed towards the support leg, with peaks occurring at contralateral toe-off (Fig. 2A). Peak lateral forces
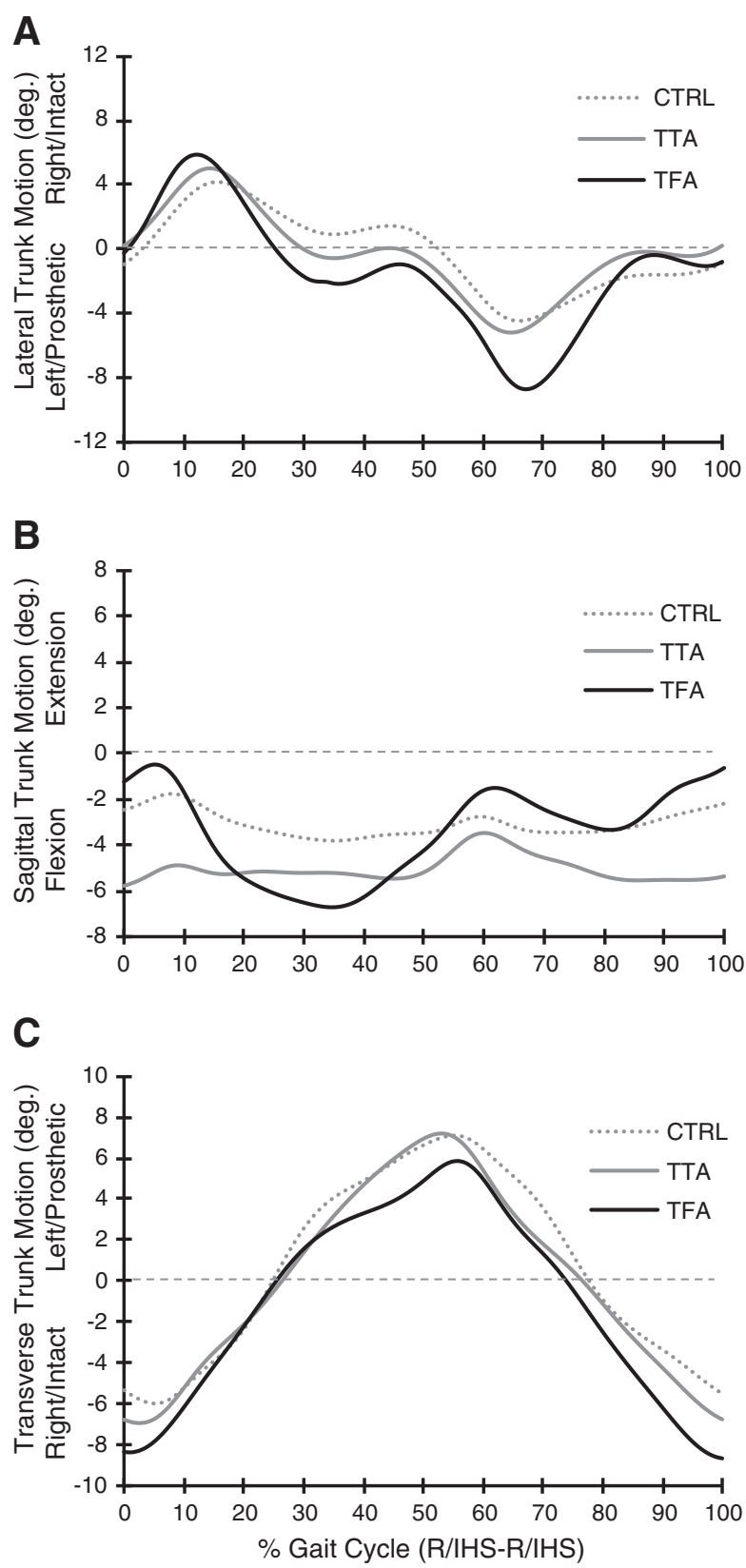

Fig. 1. Three-dimensional trunk motion (relative to pelvis). Curves represent ensemble group averages, time-normalized to one stride (right heel strike (RHS) to right heel strike for controls ("CTRL"), and intact heel strike (IHS) to intact heel strike for transtibial ("TTA") and transfemoral ("TFA") groups). (A) Lateral trunk motion. (B) Sagittal trunk motion. (C) Transverse trunk motion.

were larger $(P<0.001)$ among persons with LEA compared to controls, and were larger $(P<0.001)$ with increasing level of amputation (Table 3$)$. These were bilaterally similar among controls $(P=0.36)$ and persons with TTA $(P=0.11)$, but significantly larger $(P<0.0001)$

Table 2

Mean (SD) temporal-spatial parameters for the control (“CTRL") and lower-extremity amputation groups ("TTA": transtibial, "TFA": transfemoral).

\begin{tabular}{|c|c|c|c|c|c|c|}
\hline & \multicolumn{2}{|l|}{ CTRL } & \multicolumn{2}{|l|}{ TTA } & \multicolumn{2}{|l|}{ TFA } \\
\hline & Left & Right & Intact & Prosthetic & Intact & Prosthetic \\
\hline Step length (m) & $0.76(0.04)$ & $0.75(0.04)$ & $0.73(0.07)$ & $0.77(0.05)^{\dagger}$ & $0.71(0.05)^{*}$ & $0.78(0.06)^{\dagger}$ \\
\hline Stance duration (s) & $0.73(0.04)$ & $0.73(0.04)$ & $0.71(0.04)$ & $0.68(0.03)^{* \dagger}$ & $0.74(0.04)$ & $0.67(0.04)^{* \dagger}$ \\
\hline Swing duration (s) & $0.38(0.02)$ & $0.39(0.02)$ & $0.38(0.03)$ & $0.41(0.02)^{\dagger}$ & $0.38(0.03)$ & $0.44(0.03)^{*+}$ \\
\hline
\end{tabular}

Note: Results from post hoc contrasts are indicated by the symbols below:

* Significant $(P<0.0043)$ difference vs. CTRL.

$\dagger$ Significant $(P<0.0043)$ bilateral difference within a group. 
A

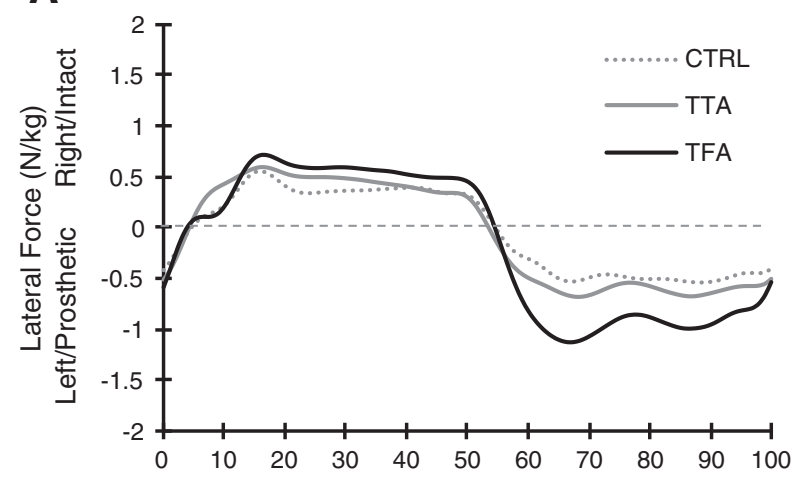

B

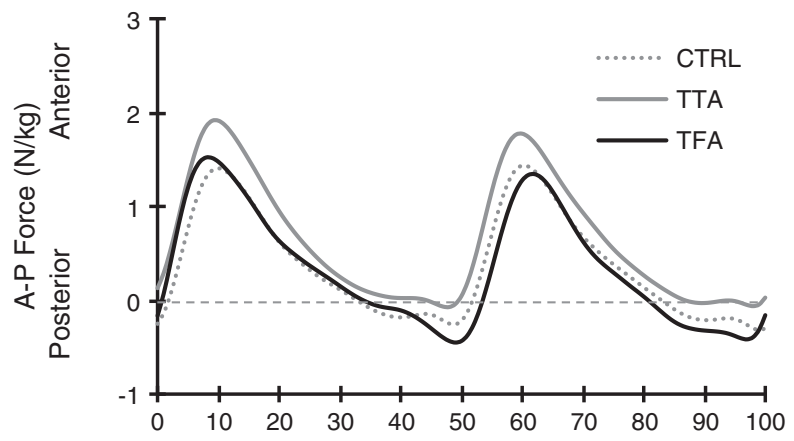

C

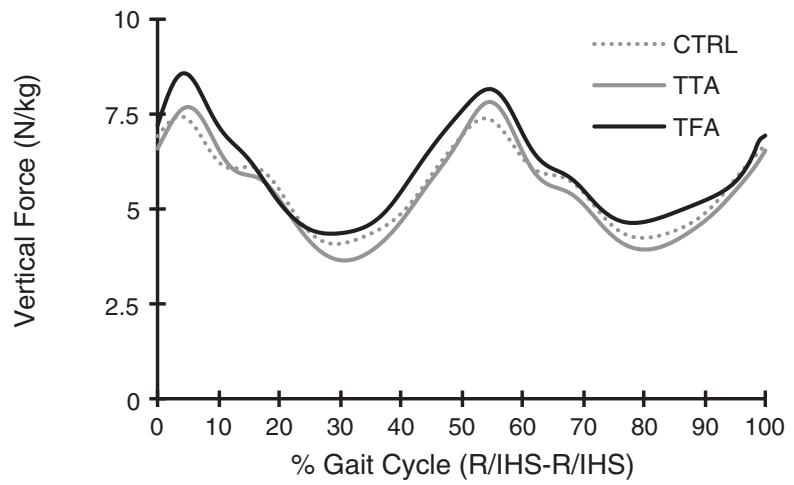

Fig. 2. Three-dimensional joint (L5/S1) reaction forces from the top-down approach (normalized by total body mass). Curves represent ensemble group averages, time-normalized to one stride (right heel strike (RHS) to right heel strike for controls ("CTRL"), and intact heel strike (IHS) to intact heel strike for transtibial ("TTA") and transfemoral ("TFA") groups). (A) Lateral reaction force. (B) Anteroposterior (A-P) reaction force. (C) Vertical reaction force (inverted).

in prosthetic vs. intact limb stance among persons with TFA (Table 3). Compared to controls, the timings of these peaks occurred sooner $(P=0.002)$ on the intact limb among persons with TTA, but were bilaterally similar $(P>0.08)$ in all groups (Table 3$)$. Anteroposterior joint reaction forces were directed posteriorly at heel strike, and quickly reversed directions as weight was shifted anteriorly toward the support leg (Fig. 2B). Peak posteriorly-directed forces were larger $(P=0.002)$ among persons with TFA vs. TTA, but these were not significantly larger $(P>0.17)$ than controls. Peak posteriorly-directed forces tended $(P=0.04)$ to be larger and occur sooner $(P=0.007)$ at prosthetic vs. intact heel strike among persons with TFA, but were bilaterally similar $(P>0.27)$ among persons with TTA and controls (Table 3). Peak anterior forces were larger $(P=0.003)$ among persons with TTA compared to controls and persons with TFA; these were bilaterally similar $(P>0.09)$ in all groups (Table 3 ). The timings of peak anterior forces tended
$(P=0.036)$ to occur sooner in persons with LEA compared to controls, but were bilaterally similar $(P>0.36)$ in all groups (Table 3$)$. Vertical joint reaction forces remained compressive throughout the gait cycle, with peaks occurring approximately coincident to the onset of singlelimb stance (Fig. 2C); these were similar between $(P>0.18)$ and within $(P>0.16)$ groups (Table 3$)$. However, relative to controls, the timings of peak vertical reaction forces occurred later $(P=0.001)$ on the intact limb among persons with TFA (Table 3).

Peak lateral bend moments were larger $(P<0.0001)$ in persons with LEA compared to controls, and were larger $(P<0.0001)$ among persons with TFA vs. TTA (Table 4$)$. These were bilaterally similar among controls $(P=0.81)$ and persons with TTA $(P=0.16)$, but were larger $(P<0.0001)$ and occurred later $(P=0.003)$ in prosthetic vs. intact stance among persons with TFA (Table 4). Peak extension moments were larger $(P=0.005)$ among persons with TTA vs. TFA and controls, but were bilaterally similar $(P>0.11)$ in all three groups (Table 4$)$. Peak extension moments occurred approximately at contralateral toe-off (Fig. 3B), and the timings were similar between $(P>0.78)$ and within $(P>0.19)$ groups (Table 4$)$. Peak axial twist moments also occurred around contralateral toe-off (Fig. $3 \mathrm{C}$ ); peak magnitudes and timings were similar between groups $(P>0.22)$ and sides $(P>0.08$; Table 4$)$.

\subsection{Top-down vs. bottom-up modeling approach}

Overall, the magnitudes of peak joint reaction forces and moments were smaller $(P<0.045)$, and the timings of these later $(P<0.026)$, from the top-down vs. bottom-up approach (see Tables 3 and 4). Regarding the peak forces and moments, these differences between model approaches were similar between and within groups, as indicated by non-significant group $\times$ model approach $(P>0.11)$ and side $\times$ model approach $(P>0.14)$ interaction effects from the ANOVA, with one exception: peak posterior reaction forces in the TFA group were larger $(P=0.013)$ from the top-down vs. bottom-up approach. For the timing of these peaks, there were two significant $(P<0.002)$ model approach $\times$ group interactions. First, the timing of peak lateral reaction forces from the top-down vs. bottom-up approach occurred later among controls and participants with TFA, but were similar among participants with TTA, and second, the timing of peak anterior reaction forces from the top-down vs. bottom-up approach occurred sooner in the TTA and TFA groups, but later among controls (Table 3).

\section{Discussion}

This study assessed resultant joint loads at the low back (L5/S1) in persons with LEA during over-ground walking, and secondarily, compared the magnitude and timing of these between bottom-up and top-down approaches. Loads at the low back have been investigated using a variety of modeling techniques, and during a variety of tasks. As such, there is a range of reported values (Callaghan et al., 1999; Cappozzo, 1983; Khoo et al., 1995; Kingma et al., 2001; Seay et al., 2008). Of note, the values presented here, which represent the reaction forces and moments from linked-segment modeling, are in reasonable agreement with previous work assessing these during over-ground walking in able-bodied individuals (Callaghan et al., 1999; Cappozzo, 1983).

\subsection{Frontal plane}

Relatively more active muscle control in the mediolateral than the anteroposterior direction is required to maintain balance during walking (e.g., O'Connor and Kuo, 2009). Specifically, balance of the trunk and pelvis over the supporting hip in single-limb stance requires a hip abduction moment to reduce pelvic drop and counteract the destabilizing laterally-directed gravitational forces (MacKinnon and Winter, 1993). For persons with LEA, increased trunk lateral flexion toward the prosthetic side has been suggested to help stabilize the body 
Table 3

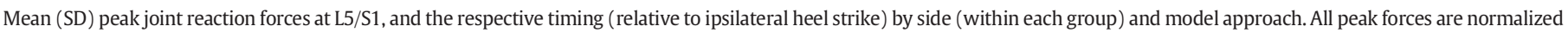
by body mass. $\mathrm{CTRL}=$ control, TTA $=$ transtibial, and TFA $=$ transfemoral.

\begin{tabular}{|c|c|c|c|c|c|c|}
\hline & \multicolumn{2}{|l|}{ CTRL } & \multicolumn{2}{|l|}{ TTA } & \multicolumn{2}{|l|}{ TFA } \\
\hline & Left & Right & Intact & Prosthetic & Intact & Prosthetic \\
\hline \multicolumn{7}{|c|}{ Top-down approach } \\
\hline \multicolumn{7}{|c|}{ Lateral } \\
\hline Peak (N/kg) & $0.50(0.17)$ & $0.57(0.15)$ & $0.64(0.35)$ & $0.81(0.24)^{*}$ & $0.70(0.31)$ & $1.21(0.35)^{* \dagger \S}$ \\
\hline Timing (ms) & $187.3(48.6)$ & $176.6(20.1)$ & $140.4(40.8) *$ & $166.2(48.9)$ & $157.6(44.1)$ & $159.8(57.4)$ \\
\hline \multicolumn{7}{|l|}{ Posterior } \\
\hline Peak (N/kg) & $0.31(0.34)$ & $0.25(0.27)$ & $0.09(0.47)$ & $0.10(0.44)$ & $0.46(0.37)$ & $0.59(0.59)^{\S}$ \\
\hline Timing (ms) & $20.1(50.8)$ & $26.8(22.8)$ & $65.5(30.5)$ & $30.1(34.8)^{\dagger}$ & $67.8(40.1)$ & $20.2(58.6)^{\dagger}$ \\
\hline \multicolumn{7}{|l|}{ Anterior } \\
\hline Peak (N/kg) & $1.46(0.39)$ & $1.42(0.40)$ & $1.93(0.64)^{*}$ & $1.81(0.49)$ & $1.56(0.60)$ & $1.39(0.56)$ \\
\hline Timing (ms) & $134.5(37.5)$ & $118.9(16.8)$ & $111.2(17.4)$ & $116.2(25.1)$ & $106.6(26.1)$ & $114.6(55.9)$ \\
\hline \multicolumn{7}{|l|}{ Vertical } \\
\hline Peak (N/kg) & $7.40(0.86)$ & $7.40(0.87)$ & $7.67(1.62)$ & $7.83(1.75)$ & $8.44(1.38)^{*}$ & $8.22(1.17)$ \\
\hline Timing (ms) & $51.5(24.9)$ & $48.8(11.5)$ & $59.7(10.6)$ & $57.2(21.7)$ & $76.4(28.7)^{*}$ & $54.2(50.1)$ \\
\hline \multicolumn{7}{|c|}{ Bottom-up approach } \\
\hline \multicolumn{7}{|c|}{ Lateral } \\
\hline Peak (N/kg) & $0.53(0.19)$ & $0.59(0.18)$ & $0.67(0.24)$ & $0.88(0.35)^{*}$ & $0.71(0.25)$ & $1.41(0.31)^{*+\S}$ \\
\hline Timing (ms) & $144.4(38.4)$ & $133.6(36.1)$ & $140.5(35.0)$ & $165.3(37.0)$ & $127.3(36.8)$ & $150.6(58.9)$ \\
\hline \multicolumn{7}{|l|}{ Posterior } \\
\hline Peak (N/kg) & $0.45(0.35)$ & $0.43(0.39)$ & $0.10(0.45)^{*}$ & $0.28(0.35)$ & $0.13(0.55)$ & $0.70(0.54)^{\dagger \S}$ \\
\hline Timing (ms) & $34.2(29.6)$ & $30.0(10.1)$ & $32.6(6.8)$ & $12.9(18.0)$ & $27.6(8.8)$ & $14.2(46.8)$ \\
\hline \multicolumn{7}{|l|}{ Anterior } \\
\hline Peak (N/kg) & $1.57(0.38)$ & $1.52(0.41)$ & $2.07(0.73)^{*}$ & $1.82(0.74)$ & $1.69(0.58)$ & $1.40(0.62)$ \\
\hline Timing (ms) & $110.9(19.5)$ & $120.8(18.8)$ & $136.3(24.4)$ & $146.1(32.1)$ & $121.2(23.8)$ & $144.8(54.9)$ \\
\hline \multicolumn{7}{|l|}{ Vertical } \\
\hline Peak (N/kg) & $8.07(0.61)$ & $8.14(0.57)$ & $8.48(1.25)$ & $8.58(1.14)$ & $8.67(1.38)$ & $8.45(1.30)$ \\
\hline Timing (ms) & $62.2(31.9)$ & $64.0(16.5)$ & $73.5(14.4)$ & $73.9(21.9)$ & $80.8(30.3)$ & $59.3(42.4)^{\dagger}$ \\
\hline
\end{tabular}

Note: Results from post hoc contrasts (within each model approach) are indicated by the symbols below:

* Significant $(P<0.0043)$ difference vs. CTRL.

$\dagger$ Significant $(P<0.0043)$ bilateral difference within a group.

$\S$ Significant $(P<0.0043)$ difference between TFA $A_{\text {prosthetic }}$ Vs. TTA $A_{\text {prosthetic }}$ and TFA intact Vs. TTA $A_{\text {intact. }}$

(Michaud et al., 2000; Tura et al., 2010), to compensate for reduced hip abduction moments due to weak, altered, or missing musculature in the residual limb (Rueda et al., 2013). Such a strategy is further supported by Mündermann et al. (2008), who reported substantial reductions in external knee and hip adduction moments with increased mediolateral trunk sway. Such lateral trunk movements, however, seem to contribute to the larger laterally-directed joint reaction forces and lateral bend moments observed in persons with LEA. Larger trunk lateral flexion with LEA found here is consistent with prior work (GoujonPillet et al., 2008; Jaegers et al., 1995), and which had poor-good correlations with peak lateral bending moments $\left(R^{2}=0.29\right)$ and reaction forces $\left(R^{2}=0.58\right)$, respectively. Larger trunk motion towards the

Table 4

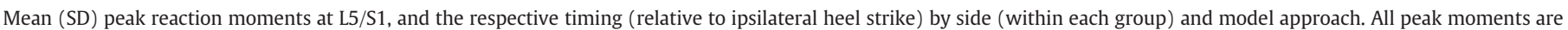
normalized by body mass $*$ stature. CTRL $=$ control, TTA $=$ transtibial, and TFA $=$ transfemoral.

\begin{tabular}{|c|c|c|c|c|c|c|}
\hline & \multicolumn{2}{|l|}{ CTRL } & \multicolumn{2}{|l|}{ TTA } & \multicolumn{2}{|l|}{ TFA } \\
\hline & Left & Right & Intact & Prosthetic & Intact & Prosthetic \\
\hline \multicolumn{7}{|l|}{ Top-down approach } \\
\hline \multicolumn{7}{|l|}{ Lateral bend } \\
\hline Peak $(\mathrm{Nm} /(\mathrm{kg} * \mathrm{~m}))$ & $0.09(0.04)$ & $0.09(0.05)$ & $0.14(0.06)$ & $0.17(0.08)^{*}$ & $0.14(0.05)$ & $0.22(0.07)^{* \dagger}$ \\
\hline Timing (ms) & $107.7(42.5)$ & $112.8(46.2)$ & $136.8(25.9)$ & $149.2(38.8)^{* \dagger}$ & $130.0(49.0)$ & $160.5(58.9)^{* \dagger}$ \\
\hline \multicolumn{7}{|l|}{ Extension } \\
\hline Peak $(\mathrm{Nm} /(\mathrm{kg} * \mathrm{~m}))$ & $0.20(0.07)$ & $0.20(0.07)$ & $0.30(0.10)^{*}$ & $0.30(0.13)^{*}$ & $0.23(0.10)$ & $0.20(0.09)^{\S}$ \\
\hline Timing (ms) & $132.2(34.3)$ & $126.2(16.6)$ & $123.6(12.5)$ & $130.4(25.1)$ & $115.9(20.3)$ & $134.1(57.6)$ \\
\hline \multicolumn{7}{|l|}{ Axial twist } \\
\hline Peak $(\mathrm{Nm} /(\mathrm{kg} * \mathrm{~m}))$ & $0.04(0.01)$ & $0.05(0.02)$ & $0.05(0.02)$ & $0.06(0.02)$ & $0.07(0.02)$ & $0.07(0.02)$ \\
\hline Timing (ms) & $116.0(56.2)$ & $106.9(25.3)$ & $107.7(42.2)$ & $89.0(75.2)$ & $100.9(26.8)$ & $125.7(51.3)$ \\
\hline \multicolumn{7}{|l|}{ Bottom-up approach } \\
\hline \multicolumn{7}{|l|}{ Lateral bend } \\
\hline Peak $(\mathrm{Nm} /(\mathrm{kg} * \mathrm{~m}))$ & $0.09(0.05)$ & $0.11(0.05)$ & $0.14(0.03)$ & $0.18(0.06)^{*}$ & $0.18(0.08)^{*}$ & $0.25(0.09)^{*+\varepsilon}$ \\
\hline Timing (ms) & $88.8(39.0)$ & $81.2(31.4)$ & $98.7(32.7)$ & $144.8(46.6)^{* \dagger}$ & $70.5(37.2)$ & $149.6(62.6)^{*+}$ \\
\hline \multicolumn{7}{|l|}{ Extension } \\
\hline Peak $(\mathrm{Nm} /(\mathrm{kg} * \mathrm{~m}))$ & $0.19(0.06)$ & $0.21(0.10)$ & $0.39(0.08)^{*}$ & $0.38(0.10)^{*}$ & $0.26(0.13)^{\S}$ & $0.27(0.12)^{\S}$ \\
\hline Timing (ms) & $66.3(46.7)$ & $78.0(20.1)$ & $81.2(42.7)$ & $116.5(38.8)^{*}$ & $107.2(43.3)^{*}$ & $94.6(59.8)$ \\
\hline \multicolumn{7}{|l|}{ Axial twist } \\
\hline Peak $(\mathrm{Nm} /(\mathrm{kg} * \mathrm{~m}))$ & $0.06(0.03)$ & $0.06(0.02)$ & $0.05(0.02)$ & $0.06(0.02)$ & $0.08(0.04)$ & $0.07(0.03)$ \\
\hline Timing (ms) & $73.4(28.2)$ & $73.4(27.7)$ & $61.8(21.8)$ & $85.7(44.3)$ & $54.6(22.7)$ & $70.9(46.7)$ \\
\hline
\end{tabular}

Note: Results from post hoc contrasts (within each model approach) are indicated by the symbols below:

* Significant $(P<0.0043)$ difference vs. CTRL.

$\dagger$ Significant $(P<0.0043)$ bilateral difference within a group.

$\S$ Significant $(P<0.0043)$ difference between TFA prosthetic Vs. TTA $A_{\text {prosthetic }}$ and TFA intact Vs. TTA $A_{\text {intact. }}$ 

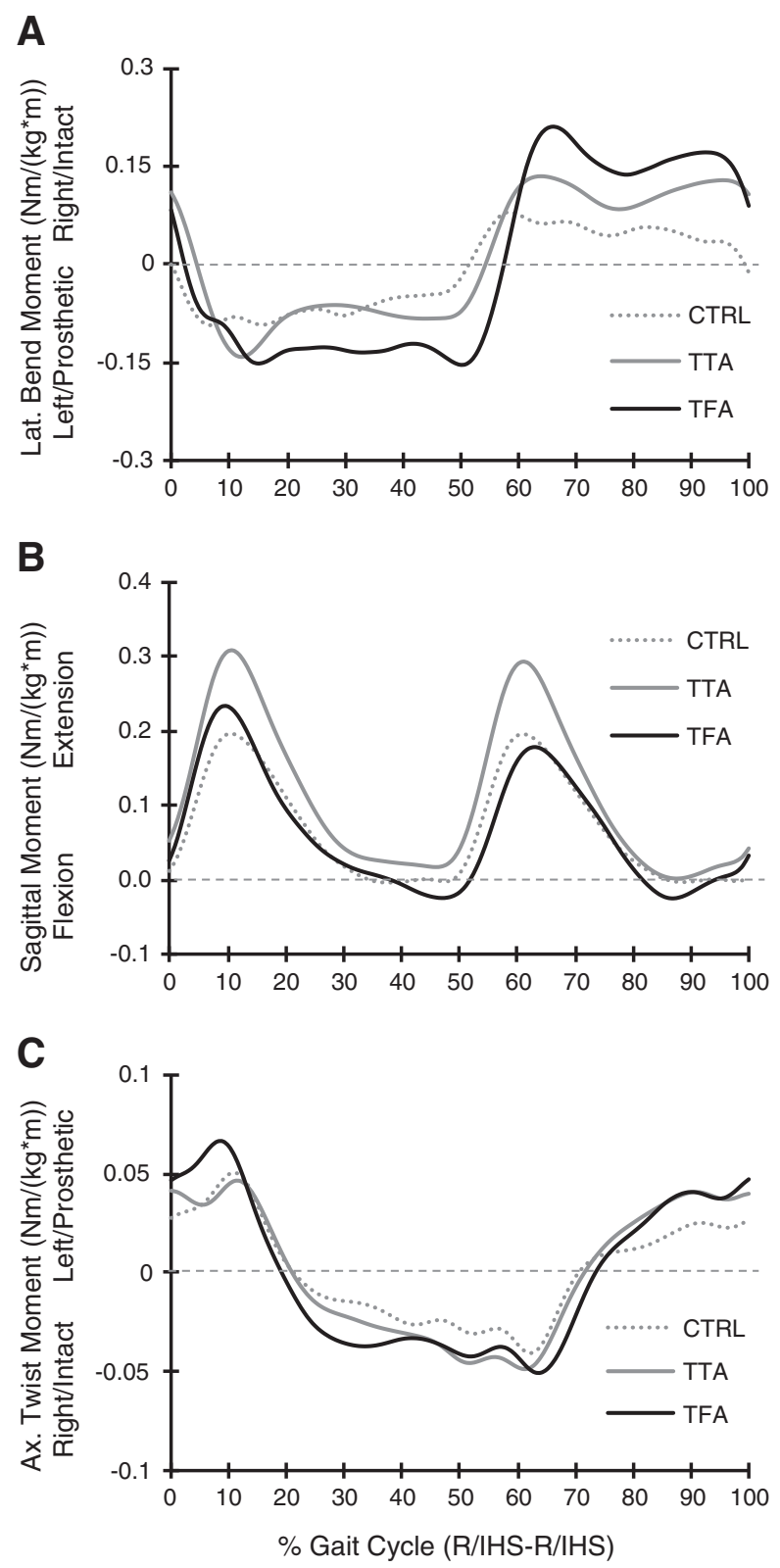

Fig. 3. Three-dimensional joint (L5/S1) reaction moments from the top-down approach (normalized by total body mass $*$ stature). Curves represent ensemble group averages, time-normalized to one stride (right heel strike (RHS) to right heel strike for controls ("CTRL"), and intact heel strike (IHS) to intact heel strike for transtibial ("TTA") and transfemoral ("TFA") groups). (A) Lateral bend moment. (B) Flexion-extension moment. (C) Axial twist moment. Note that "internal" moments are illustrated (by inversion of calculated external moments).

prosthetic limb among persons with unilateral LEA likely also results in asymmetric muscular demands, as indicated here by the larger internal moments on the intact side during prosthetic stance (Fig. 3A). Asymmetrical motion and loading in tissues in/surrounding the low back have been associated with an increased risk for LBP (Davis and Marras, 2000), and may contribute to previously identified asymmetric trunk mechanical properties with unilateral LEA during multidirectional trunk perturbations (Hendershot et al., 2013).

\subsection{Sagittal plane}

Larger trunk motions in the sagittal plane and increased trunk forward lean have been observed in persons with LEA (Goujon-Pillet et al., 2008). These alterations in trunk motion/posture can assist with weight-bearing balance and forward progression of the body, compensating for a lack of power generation at the ankle. Additionally, trunk forward lean can also facilitate hip extension, a movement that often is limited by hip flexion contractures or reduced iliopsoas flexibility among persons with LEA (Gailey et al., 2008). However, trunk flexed postures place additional demand on trunk and hip extensor musculature, as evidenced here by the larger internal extension moments at L5/S1 during gait among persons with LEA (Fig. 3B). Larger extensor moments among persons with TTA, but not with TFA, may indicate that persons with TFA adopt more lordotic trunk postures to accommodate changes in hip orientation and/or trunk-pelvic alignment. However, lumbar spinal alignment could not be elucidated with the current marker setup. Larger posteriorly directed reaction forces at prosthetic vs. intact heel strike among persons with TFA likely result from a significantly longer step with the prosthetic limb, and which occurred coincident with peak trunk extension (Fig. 1B).

\subsection{Transverse plane}

As compared to anatomical range-of-motion, the relative rotation between the shoulder girdle and pelvis during gait is typically largest in the transverse plane. Therefore, it is often hypothesized that control of transverse trunk/spine rotations play an important role in LBP prevention. For example, the mechanical coupling between trunk and pelvis rotations in the transverse plane during gait is higher in able-bodied individuals with LBP (van den Hoorn et al., 2012). Here, no significant differences were observed in axial twist moments at the low back between persons with/without LEA. Persons with TFA walk with reduced counter-rotation of the trunk and pelvis (Goujon-Pillet et al., 2008), which has been related to increased axial stiffness or "guarding" behavior (Selles et al., 2001). More in-phase rotations between the trunk and pelvis with LEA would seem to reduce the transmission of torque between the upper and lower body among persons with LEA (LaFiandra et al., 2002). In contrast, coordination of the trunk and pelvis in the transverse plane in persons with transfemoral amputation may be altered with current LBP (Morgenroth et al., 2010), but associated changes in joint kinetics were not reported as part of that study. The coordination and loading between the trunk and pelvis in the transverse plane is largely influenced by walking speed and arm swing (Bruijn et al., 2008), and it is therefore possible that faster (or slower) walking speeds may better discriminate differences in joint reaction moments with LEA in the transverse plane.

\subsection{Top-down vs. bottom-up approach}

Larger reaction forces and moments from the bottom-up vs. topdown approach are in agreement with previous literature, and which have been suggested to result from impulsive contributions arising from the foot-ground impact (Callaghan et al., 1999; Cappozzo, 1984; Seay et al., 2008). When comparing these two approaches, it has been speculated that the bottom-up analysis is a better approach as the trunk segment, which is often oversimplified as a single rigid segment, is excluded in bottom-up models (Kingma et al., 1996). Conversely, reactive joint loads estimated from bottom-up analyses are substantially influenced by the accuracy of the location of application, and the rigidbody assumptions regarding the transmission, of the forces arising from foot-ground impacts through the body (Kingma et al., 1996; Lafortune et al., 1996). These factors may be more important when studying persons with LEA, where there exists a (bilateral) difference in lower-extremity soft tissues and altered mechanical properties of prosthetic components vs. physiologic tissues that may influence the actual vs. modeled transmission of forces to the low back. Of note, here we modeled the anthropometric/inertial properties of the residual and prosthetic limbs using parameters identical to participants' intact limbs. However, since peak kinetic values were extracted for group and limb comparisons, and which here occurred primarily during the 
stance phase, such differences in lower-extremity inertial parameters (for bottom-up analyses) are likely negligible, as external loads (ground reaction forces) dominate the kinetic calculations (Baum et al., 2013). Regardless, the overall trends in peak forces and moments between and within (bilateral) groups observed here were similar between approaches; though, some interactions (between groups and approaches) were noted in the timing of these peaks.

\subsection{Limitations}

Participants in the current study consisted of young and active military personnel with traumatic amputations. Therefore, the results may not be generalizable to all persons with LEA, including older, less active individuals or those with other amputation etiologies. Also, whether participants had a history of LBP was not controlled in the present study, as there was insufficient documentation for reliably stratifying participants based on this factor. However, participants who had indicated current LBP at the time of the gait evaluation were not included in these analyses. Comparisons between persons with LEA with/without current or a history of LBP would be of interest in future work, to help determine altered lumbar loading as a potential causative factor vs. effect of LBP among persons with LEA. Finally, this study calculated lumbosacral joint reaction forces and moments, irrespective of individual trunk muscle contributions. Forces arising from trunk muscle (co)activity can substantially increase spinal loads (Granata and Marras, 1995), and bone-bone spinal compressive forces during walking in ablebodied individuals calculated using an electromyography (EMG)-driven model were $\sim 3$ times larger compared to linked-segment analyses (Callaghan et al., 1999). Observed increases in trunk kinematics among persons with LEA may alter trunk muscle recruitment patterns and increase demands on trunk musculature to maintain equilibrium and stability of the spine, and which thereby may require increased trunk muscle coactivity (i.e., larger muscle forces) during gait and movement. As such, future work should incorporate trunk muscle contributions to lumbosacral joint load estimation, as these may further alter lumbosacral joint loads compared to able-bodied individuals.

\section{Conclusions}

In summary, the current findings begin to identify how alterations in trunk motion during gait secondary to LEA adversely affect loads at the low back, particularly in the frontal plane. Such evidence suggests potential mechanistic pathways through which altered trunk motion and spinal loading may contribute to low-back injury risk among persons with LEA. While these loads are of low to moderate magnitudes, repetitive exposure over time may associate altered spinal loading with LBP onset and recurrence in this population (Kumar, 2001; McGill, 2007). As such, interventions and/or rehabilitation strategies aimed at reducing trunk motion (acceleration) subsequent to LEA may play a central role in controlling or reducing the prevalence of LBP as a secondary impairment among individuals with lower-extremity amputation.

\section{Acknowledgments}

This work was supported by the Center for Rehabilitation Sciences Research (NF90UG), DoD Defense Health Program. The views expressed in this manuscript are those of the authors, and do not necessarily reflect the official policy of the Departments of the Army, Navy, Defense, nor the United States Government.

\section{References}

Baum, B.S., et al., 2013. Amputee locomotion: determining the inertial properties of running-specific prostheses. Arch. Phys. Med. Rehabil. 94, 1776-1783.
Bruijn, S.M., Meijer, O.G., van Dieën, J.H., Kingma, I., Lamoth, C.J.C., 2008. Coordination of leg swing, thorax rotations, and pelvis rotations during gait: the organisation of total body angular momentum. Gait Posture 27, 455-462.

Callaghan, J.P., Patla, A.E., McGill, S.M., 1999. Low back three-dimensional joint forces, kinematics, and kinetics during walking. Clin. Biomech. 14, 203-216.

Cappozzo, A., 1983. The forces and couples in the human trunk during level walking. J. Biomech. 16, 265-277.

Cappozzo, A., 1984. Compressive loads in the lumbar vertebral column during normal level walking. J. Orthop. Res. 1, 292-301.

Cappozzo, A., Figura, F., Gazzani, F., Leo, T., Marchetti, M., 1982. Angular displacements in the upper body of AK amputees during level walking. Prosthetics Orthot. Int. 6, 131-138.

Cappozzo, A., Gazzani, F., 1983. Spinal loading during abnormal walking. In: Huiskes, R., van Campen, D.H., de Wijn, J.R. (Eds.), Biomechanics: Principles and Applications. Springer, Netherlands, pp. 141-148.

Cheng, K., Chen, H., Chen, S., Lee, S., 1998. Influences of walking speed change on the lumbosacral joint force distribution. Biomed. Mater. Eng. 8, 155-165.

Davis, K.G., Marras, W.S., 2000. The effects of motion on trunk biomechanics. Clin. Biomech. 15, 703-717.

Ehde, D.M., Smith, D.G., Czerniecki, J.M., Campbell, K.M., Malchow, D.M., Robinson, L.R., 2001. Back pain as a secondary disability in persons with lower limb amputations. Arch. Phys. Med. Rehabil. 82, 731-734.

Gailey, R., Allen, K., Castles, J., Kucharik, J., Roeder, M., 2008. Review of secondary physical conditions associated with lower-limb amputation and long-term prosthetic use. J. Rehabil. Res. Dev. 45, 15-29.

Gillet, C., Duboy, J., Barbier, F., Armand, S., Jeddi, R., Lepoutre, F.-X., et al., 2003. Contribution of accelerated body masses to able-bodied gait. Am. J. Phys. Med. Rehabil. 82, 101-109.

Goujon-Pillet, H., Sapin, E., Fodé, P., Lavaste, F., 2008. Three-dimensional motions of trunk and pelvis during transfemoral amputee gait. Arch. Phys. Med. Rehabil. 89, 87-94.

Granata, K.P., Marras, W.S., 1995. The influence of trunk muscle coactivity on dynamic spinal loads. Spine 20, 913-919.

Hendershot, B.D., Bazrgari, B., Nussbaum, M.A., 2013. Persons with unilateral lower-limb amputation have altered and asymmetric trunk mechanical and neuromuscular behaviors estimated using multidirectional trunk perturbations. J. Biomech. 46, 1907-1912.

Iino, Y., Kojima, T., 2012. Validity of top-down approach of inverse dynamics analysis in fast and large rotational trunk movements. J. Appl. Biomech. 28, 420-430.

Jaegers, S.M.H.J., Arendzen, J.H., de Jongh, H.J., 1995. Prosthetic gait of unilateral transfemoral amputees: a kinematic study. Arch. Phys. Med. Rehabil. 76, 736-743.

Khoo, B.C.C., Goh, J.C.H., Bose, K., 1995. A biomechanical model to determine lumbosacral loads during single stance phase in normal gait. Med. Eng. Phys. 17, 27-35.

Kinzel, G.L., Hall, A.S., Hillberry, B.M., 1972. Measurement of the total motion between two body segments -I analytical development. J. Biomech. 5, 93-105.

Kingma, I., Baten, C.T.M., Dolan, P., Toussaint, H.M., van Dieën, J.H., de Looze, M.P., et al., 2001. Lumbar loading during lifting: a comparative study of three measurement techniques. J. Electromyogr. Kinesiol. 11, 337-345.

Kingma, I., de Looze, M.P., Toussaint, H.M., Klijnsma, H.G., Bruijnen, T.B.M., 1996. Validation of a full body 3-D dynamic linked segment model. Hum. Mov. Sci. 15, 833-860.

Kumar, S., 2001. Theories of musculoskeletal injury causation. Ergonomics 44, 17-47.

LaFiandra, M., Holt, K.G., Wagenaar, R.C., Obusek, J.P., 2002. Transverse plane kinetics during treadmill walking with and without a load. Clin. Biomech. 17, 116-122.

Lafortune, M.A., Lake, M.J., Hennig, E.M., 1996. Differential shock transmission response of the human body to impact severity and lower limb posture. J. Biomech. 29, 1531-1537.

MacKinnon, C.D., Winter, D.A., 1993. Control of whole body balance in the frontal plane during human walking. J. Biomech. 26, 633-644.

McGill, S.M., 2007. Low Back Disorders: Evidence-based Prevention and Rehabilitation, 2nd ed. Human Kinetics, Champaign, IL.

Michaud, S., Gard, S., Childress, D., 2000. A preliminary investigation of pelvic obliquity patterns during gait in persons with transtibial and transfemoral amputation. J. Rehabil. Res. Dev. 37, 1-10.

Morgenroth, D.C., Orendurff, M.S., Shakir, A., Segal, A., Shofer, J., Czerniecki, J.M., 2010. The relationship between lumbar spine kinematics during gait and low-back pain in transfemoral amputees. Am. J. Phys. Med. Rehabil. 89, 635-643.

Mündermann, A., Asay, J.L., Mündermann, L., Andriacchi, T.P., 2008. Implications of increased medio-lateral trunk sway for ambulatory mechanics. J. Biomech. 41, $165-170$.

O'Connor, S.M., Kuo, A.D., 2009. Direction-dependent control of balance during walking and standing. J. Neurophysiol. 102, 1411-1419.

Plamondon, A., Gagnon, M., Desjardins, P., 1996. Validation of two 3-D segment models to calculate the net reaction forces and moments at the L5S1 joint in lifting. Clin. Biomech. 11, 101-110.

Reed, M.P., Manary, M.A., Schneider, L.W., 1999. Method for measuring and representing automobile occupant posture. SAE Technical Paper Series 199901-0959, pp. 1-15.

Reiber, G.E., McFarland, L.V., Hubbard, S., Maynard, C., Blough, D.K., Gambel, J.M., et al., 2010. Servicemembers and veterans with major traumatic limb loss from Vietnam war and OIF/OEF conflicts: Survey methods, participants, and summary findings. J. Rehabil. Res. Dev. 47, 275-298.

Riemer, R., Hsiao-Wecksler, E.T., Zhang, X., 2008. Uncertainties in inverse dynamics solutions: a comprehensive analysis and an application to gait. Gait Posture 27, 578-588.

Rueda, F.M., Diego, I.M.A., Sánchez, A.M., Tejada, M.C., Montero, F.M.R., Page, J.C.M., 2013. Knee and hip internal moments and upper-body kinematics in the frontal plane in unilateral transtibial amputees. Gait Posture 37, 436-439. 
Sagawa Jr., Y., Turcot, K., Armand, S., Thevenon, A., Vuillerme, N., Watelain, E., 2011. Biomechanics and physiological parameters during gait in lower-limb amputees: a systematic review. Gait Posture 33, 511-526.

Seay, J., Selbie, W.S., Hamill, J., 2008. In vivo lumbo-sacral forces and moments during constant speed running at different stride lengths. J. Sports Sci. 26, 1519-1529.

Selles, R.W., Wagenaar, R.C., Smit, T.H., Wuisman, P.I.J.M., 2001. Disorders in trunk rotation during walking in patients with low back pain: a dynamical systems approach. Clin. Biomech. 16, 175-181.

Taghipour, H., Moharamzad, Y., Mafi, A.R., Amini, A., Naghizadeh, M.M., Soroush, M.R., et al., 2009. Quality of life among veterans with war-related unilateral lower extremity amputation: a long-term survey in a prosthesis center in Iran. J. Orthop. Trauma 23, 525-530.
Tura, A., Raggi, M., Rocchi, L., Cutti, A.G., Chiari, L., 2010. Gait symmetry and regularity in transfemoral amputees assessed by trunk accelerations. Journal of NeuroEngineering and Rehabilitation. , 7, pp. 1-10.

van den Hoorn, W., Bruijn, S.M., Meijer, O.G., Hodges, P.W., van Dieën, J.H., 2012. Mechanical coupling between transverse plane pelvis and thorax rotations during gait is higher in people with low back pain. J. Biomech. 45, 342-347.

Winter, D.A., 1990. Biomechanics and Motor Control of Human Movement, 2nd Ed. Wiley Interscience, New York, NY.

Ziegler-Graham, K., MacKenzie, E.J., Ephraim, P.L., Travison, T.G., Brookmeyer, R., 2008 Estimating the prevalence of limb loss in the United States: 2005 to 2050. Arch. Phys. Med. Rehabil. 89, 422-429. 